\title{
The SNAP-Ed Evaluation Framework: Nationwide Uptake and Implications for Nutrition Education Practice, Policy, and Research
}

\author{
Jini E. Puma, $\mathrm{PhD}^{1}$; Max Young, MPH, RD ${ }^{2}$; Susan Foerster, MPH, RD ${ }^{3}$; \\ Kimberly Keller, $\mathrm{PhD}^{4}$; Pamela Bruno, $\mathrm{MPH}^{5}$; Karen Franck, $\mathrm{PhD}^{6}$; Andy Naja-Riese, $\mathrm{MSPH}^{7}$
}

\begin{abstract}
Objective: Provide the first baseline census of Supplemental Nutrition Assistance Program Education (SNAPEd) state implementing agencies' (SIAs) intent to use and evaluate 51 indicators described in the SNAP-Ed Evaluation Framework.

Methods: A cross-sectional study design was used to administer electronic surveys to 124 SIAs who received SNAP-Ed funding in 2017. Descriptive statistics were used to analyze the results.

Results: Of 51 indicators, SIAs reported their intent to impact an average of 19 indicators and evaluate an average of 12. More SIAs reported the intention to impact indicators at the individual (59\%) and environmental levels (48\%), compared with the sectors of influence (20\%) and population levels $(30 \%)$ of the framework. In addition, more SIAs intended to impact and evaluate short- or medium-term indicators, compared with long-term indicators.

Conclusions and Implications: These findings illustrate the progress made toward aggregating metrics to measure the collective impact of SNAP-Ed.

Key Words: food security, evaluation, nutrition education, SNAP-Ed, policy (J Nutr Educ Behav. 2021;53:336-342.)
\end{abstract}

Accepted October 24, 2020. Published online December 11, 2020.

\section{INTRODUCTION}

\section{Supplemental Nutrition Assistance \\ Program Education}

Supplemental Nutrition Assistance Program Education (SNAP-Ed) is funded through the Nutrition Education and Obesity Prevention Grant Program in the Healthy Hunger-Free Kids Act of 2010. ${ }^{1}$ Supplemental Nutrition Assistance Program Education is charged with improving the likelihood that people with incomes at or below $185 \%$ of the federal poverty level, especially those residing in communities with a significant low-income population, will make healthy food choices within a limited budget and choose physically active lifestyles consistent with the Dietary Guidelines for Americans. ${ }^{1,2}$ This includes at least $25 \%$ of the US population or well over

\footnotetext{
${ }^{1}$ Rocky Mountain Prevention Research Center, Department of Community and Behavioral Health, Colorado School of Public Health, University of Colorado Denver, Denver, CO

${ }^{2}$ Colorado Department of Human Services, Denver, CO

${ }^{3}$ Network for a Healthy California, California Department of Public Health, Sacramento, CA ${ }^{4}$ Department of Nutrition and Exercise Physiology, University of Missouri, Columbia, MO

${ }^{5}$ Center for Excellence in Public Health, University of New England, Portland, ME

${ }^{6}$ Institute of Agriculture, University of Tennessee, Knoxville, TN

${ }^{7}$ Agricultural Institute of Marin, San Rafael, CA

Conflict of Interest Disclosure: The authors have not stated any conflicts of interest.

Address for correspondence: Jini E. Puma, PhD, Rocky Mountain Prevention Research Center, Department of Community and Behavioral Health, Colorado School of Public Health, University of Colorado Denver, 13001 East 17th Ave, Campus Box B-119, Denver, CO 80045; E-mail: jini.puma@cuanschutz.edu

(C) 2020 The Authors. Published by Elsevier Inc. on behalf of Society for Nutrition Education and Behavior. This is an open access article under the CC BY-NC-ND license (http:// creativecommons.org/licenses/by-nc-nd/4.0/)

https://doi.org/10.1016/j.jneb.2020.10.015
}

80 million people. ${ }^{3}$ The SNAP state agencies in all 50 states, the District of Columbia, Guam, and the US Virgin Islands, participate in SNAP-Ed by contracting with 136 SNAP-Ed state implementing agencies (SIAs) to conduct health promotion programming. Many SIAs subcontract with different types of public, nonprofit, and business organizations to provide direct services. Collectively, hundreds of interventions tailored to low-income Americans of different age, race/ethnicity, and linguistic segments are delivered each year in compliance with the approved state plans of the Food and Nutrition Service (FNS), an agency of the US Department of Agriculture (USDA).

Nationwide, SNAP-Ed SIAs reported conducting activities in more than 60,000 low-resource community locations in 2017 (USDA Education and Administration Reporting System, personal communications, 2017). Supplemental Nutrition Assistance Program Education programming reached 3.8 million people through direct education (interventions in which a participant is actively engaged in the 
learning process with an educator and/ or interactive media) in the fiscal year 2018, the most of any USDA nutrition education program. ${ }^{4}$ At $\$ 404$ million in 2017, SNAP-Ed is second only to the Special Supplemental Program for Women, Infants and Children in its funding for nutrition education. ${ }^{4}$

\section{Development of SNAP-Ed Evaluation Framework}

Because SNAP-Ed is designed to be flexible and state-driven to meet the specific needs of limited-resource audiences and communities, measuring SNAP-Ed's collective impact has historically been challenging. ${ }^{4}$ Starting in 2013, a selected group of states, FNS staff, and other experts worked together through the National Collaborative on Childhood Obesity Research to develop the SNAP-Ed Evaluation Framework (Framework); it was introduced nationwide with its companion interactive Interpretive Guide for the 2017 federal fiscal year. ${ }^{5,6}$ The Framework was designed to provide clear outcome indicators for each level of the Social Ecological Model, with the addition of the population level, and help SIAs track large-scale change over time. Figure 1 shows the 51 Framework outcomes from which SIAs can choose, based on their state's needs assessments, priorities, and resources. An accompanying Interpretive Guide is designed as a living document to help program planners link each outcome indicator with specific measures and evaluation tools. ${ }^{5,6}$ In 2017, FNS specified 7 priority indicators that all SIAs were encouraged to address in the first year of Framework implementation. These priority indicators included the following: individual-level indicators (medium term 1 [MT1]: healthy eating, medium term 2 [MT2]: food resource management, and medium term 3 [MT3]: physical activity and reduced sedentary behavior); environmental setting indicator (short term 7 [ST7]: organizational partnerships); sector of influence indicator (short term 8 [ST8]: multisector partnerships and planning); and population results indicator (result 2 [R2]: fruits and vegetables).

The 2017 SNAP-Ed Census (Census) was conducted to quantify the initial uptake of the Framework and showcase the breadth and depth of
SNAP-Ed programming and evaluation activity across the country. It was conducted as a collaboration of the Association of SNAP Nutrition Education Administrators (ASNNA), the University of Colorado Denver (Rocky Mountain Prevention Research Center), and the Mountain Plains Regional Office of USDA's FNS. This study was determined to be not human subject research by the Colorado Multiple Institutional Review Board (protocol no. 16-2045). The primary objective of the study was to obtain baseline information about SIAs' intent to use the Framework to impact and evaluate the 51 outcome indicators.

\section{METHODS}

\section{Participants}

The target population for the Census was the 136 unique SIAs delivering nutrition education and obesity prevention programming with federal SNAP-Ed funding. Targeted SIAs were located in all 50 states, the District of Columbia, Guam, and the US Virgin Islands. Of the targeted SIAs, 124

\section{SNAP-ED EVALUATION FRAMEWORK}

Nutrition, Physical Activity, and Obesity Prevention Indicators

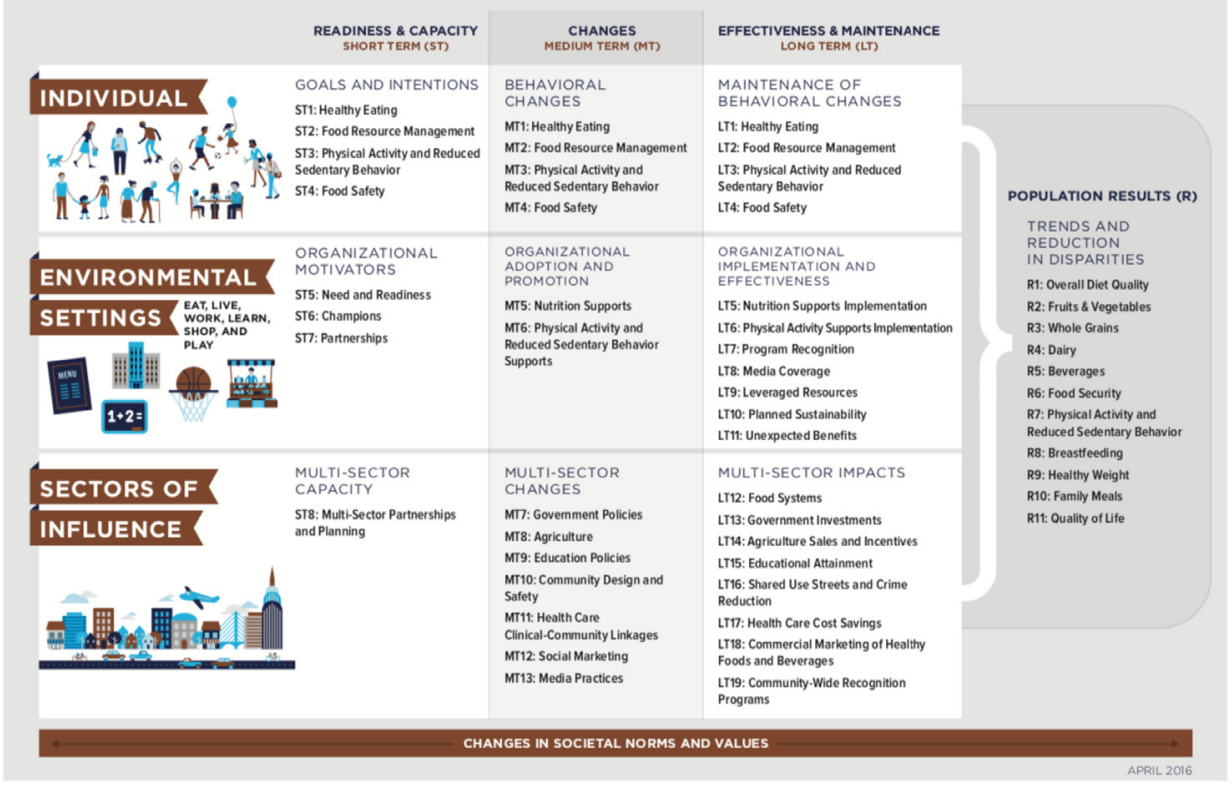

Figure 1. Supplemental Nutrition Assistance Program Education (SNAP-Ed) Evaluation Framework: nutrition, physical activity, and obesity prevention indicators. ${ }^{5}$ 
participated in the Census, with a response rate of $91 \%$.

\section{Procedure}

The names and email addresses for SIA SNAP-Ed directors were identified in the summer of 2016 in collaboration with FNS's 7 SNAP-Ed Regional Coordinators and the SNAP state agencies. The FNS Regional Coordinators and state agency staff assisted ASNNA by encouraging SIAs to participate. The SIA designees were instructed to select the person most knowledgeable about programming and evaluation to complete the Census, to use their federal fiscal year 2017 state plan, which became effective on October 1, 2016, to inform their responses, and to include the work of any local-, regional-, or statelevel subcontractors. State implementing agencies received an email with a link to REDCap (Vanderbilt University, Nashville, TN)—a secure, web-based data capture tool ${ }^{8}$ hosted at the University of Colorado Denver for the Census. The data collection procedure then followed the Tailored Design Method. ${ }^{9}$ Once a respondent completed the Census, a thank you note was sent immediately. For others, reminder emails were sent once a week for up to 3 subsequent weeks. If no response was received after 4 attempts, personal calls were made by peers in ASNNA offering help and urging their response. Data collection ended in late October, 2016.

\section{Instrument}

The Census survey instrument was adapted from a poll used in the FNS Mountain Plains Region to inform a meeting of its 10 states in early summer of 2016. By late summer, a team from ASNNA expanded the poll into a census for nationwide administration. The instrument was designed to retrieve information about the planned use of the new Framework and its Interpretive Guide. It was organized to include all 51 indicators in the 4 spheres of influence of the Framework: individual, environmental settings, sectors of influence, and population results. The Census asked about the SIA's intent to impact and to evaluate each outcome indicator. The response options for questions included: yes, no, and I don't know. Definitions of each indicator referenced the Interpretive Guide. Additional questions asked about SIAs' characteristics, use of the Framework and Interpretive Guide, and training and technical needs (not included in this article). There was a total of 115 questions on the survey instrument. The Census instrument was pilottested before it was administered nationally.

\section{Data Analysis}

All quantitative data were exported from REDCap into SPSS (version 24.0, IBM Corp, Armonk, NY, 2016) for analysis. Basic descriptive statistics (ie, frequencies, means, and SDs) were computed. In addition, the mean percent gap in SIAs' intent to impact and evaluate each indicator was calculated by subtracting the percent of SIAs evaluating each indicator from the percent of SIAs intending to impact each indicator to get a percentage point difference for each indicator (gap in evaluation). This value was then averaged across all 51 indicators (results included in the Results section).

\section{RESULTS}

\section{SIA Characteristics}

At least 1 SIA response was received from every state and the District of Columbia. Results do not include the territories of Guam, the US Virgin Islands, or 4 of the 7 Indian Tribal Organizations (ITOs) serving as SIAs. On investigation, it was learned that the 4 missing ITOs were awarded SNAP-Ed funding immediately before the administration of the Census, possibly hindering their response. In total, 75 of 85 SIAs ( $88 \%$ ) located in the 28 states that ranked in the top 10 highest for the prevalence of obesity, food insecurity, and/or proportion of low-income residents responded to the Census.

The majority of SIAs were university-based (54\%); 41\% worked in USDA Cooperative Extension agencies at land grant universities. Another 23\% were from nonprofit organizations (eg, faith-based, public health, or food banks), about 15\% from state or local governments (especially public health departments), and about 6\% from ITOs. Most had delivered SNAP-Ed for many years. Nearly two-thirds began offering SNAP-Ed before 2011 when they were required to identify state share that would qualify for Federal Financial Participation (matching dollars). The remaining 29\% entered after 2011 when grants replaced state match, and the scope of activities expanded from direct education and social marketing to include multilevel and public health approaches.

\section{Intent to Impact and Evaluate Framework Indicators}

Most of the SIAs targeted the priority indicators from the individual and environmental settings spheres; only half targeted priority indicators from the sectors of influence and population spheres. Considering all 51 indicators, SIAs reported intending to impact an average of 19 indicators $(\mathrm{SD}=11$ indicators), of which they planned to evaluate an average of 12 indicators ( $\mathrm{SD}=7$ indicators). For the 51 indicators, the mean gap was $15 \%$ between intent to impact and evaluate (range 3\%-30\%).

In general, wide variability was seen in the intent to impact compared with the planned evaluation of Framework indicators (Table). More SIAs intended to impact indicators closer to the inner spheres of influence in the Social Ecological Model: individual level (mean $=59 \%$; SD = 22\%) and environmental settings level (mean $=48 \%$; $\mathrm{SD}=23 \%$ ). Close to one-third of SIAs intended to impact outcomes leading to population results (mean $=30 \%$; $\mathrm{SD}=11 \%$ ) with the fewest agencies working at the sectors of influence level $($ mean $=20 \% ; \mathrm{SD}=12 \%)$. Most SIAs intended to address short-term indicators $\quad($ mean $=63 \% ; \quad S D=14 \%)$ and/or medium-term indicators (mean $=46 \%$; SD $=30 \%$ ), whereas a quarter planned for long-term indicators $($ mean $=26 \%$; SD $=15 \%)$. For all indicators in each sphere and time frame of the Framework, there was a gap between the intent to evaluate and the indicators that they planned 
Table. Mean Percentage of SIAs Intending to Impact and Evaluate Indicators Across the Various Sections of the Framework

\begin{tabular}{lcc} 
Section of the Framework & $\begin{array}{c}\text { Mean \% of SIAs Intending to } \\
\text { Impact Indicators Across all } \\
\text { Indicators in Section (SD), } \mathbf{n = 1 2 4}\end{array}$ & $\begin{array}{c}\text { Mean \% of SIAs Intending to } \\
\text { Evaluate Indicators Across all } \\
\text { Indicators in Section (SD), } \mathbf{n}=\mathbf{1 2 4}\end{array}$ \\
Individual level & $59(22)$ & $41(29)$ \\
Environmental setting level & $48(23)$ & $35(19)$ \\
Sectors of influence level & $20(12)$ & $10(8)$ \\
Population level & $30(11)$ & $14(7)$ \\
Short-term & $63(14)$ & $44(17)$ \\
Medium-term & $46(30)$ & $35(30)$ \\
Long-term & $26(15)$ & $13(9)$ \\
\hline
\end{tabular}

SIA indicates state implementing agency.

to impact (gap range $=4 \%-24 \%$; mean gap $=14 \%)$.

Figure 2 displays results for each of the 4 spheres of influence in the Framework. For the individual, environmental settings, and sectors of influence spheres, indicators generally move from short- to long-term. The percentage of SIAs that intended to impact an indicator is shown in blue, whereas orange bars represent the percentage of SIAs that planned to evaluate the indicator.

\section{Individual Level}

Individual behavior change outcomes are generally defined as pre-post changes resulting from direct education, often as series-based classes. Longterm outcomes are defined as those persisting for 6 months or more., 6 Figure 2 shows that the percent of SIAs intending to impact the indicators ranged from $22 \%$ to $95 \%$, and intent to evaluate ranged from $3 \%$ to $90 \%$. Most intended to work on medium- term individual behavior change indicators, ranging from $75 \%$ to $95 \%$ of all SIAs planning to impact and $65 \%$ to $90 \%$ planning to evaluate them. Very high percentages of SIAs intended to impact and evaluate the 3 mediumterm indicators named by FNS as priority indicators: healthy eating (impact $=95 \%$, evaluate $=90 \%$, , physical activity and sedentary behavior (impact $=79 \%$, evaluate $=70 \%$, and food resource management (impact $=$ $76 \%$, evaluate $=65 \%$ ).

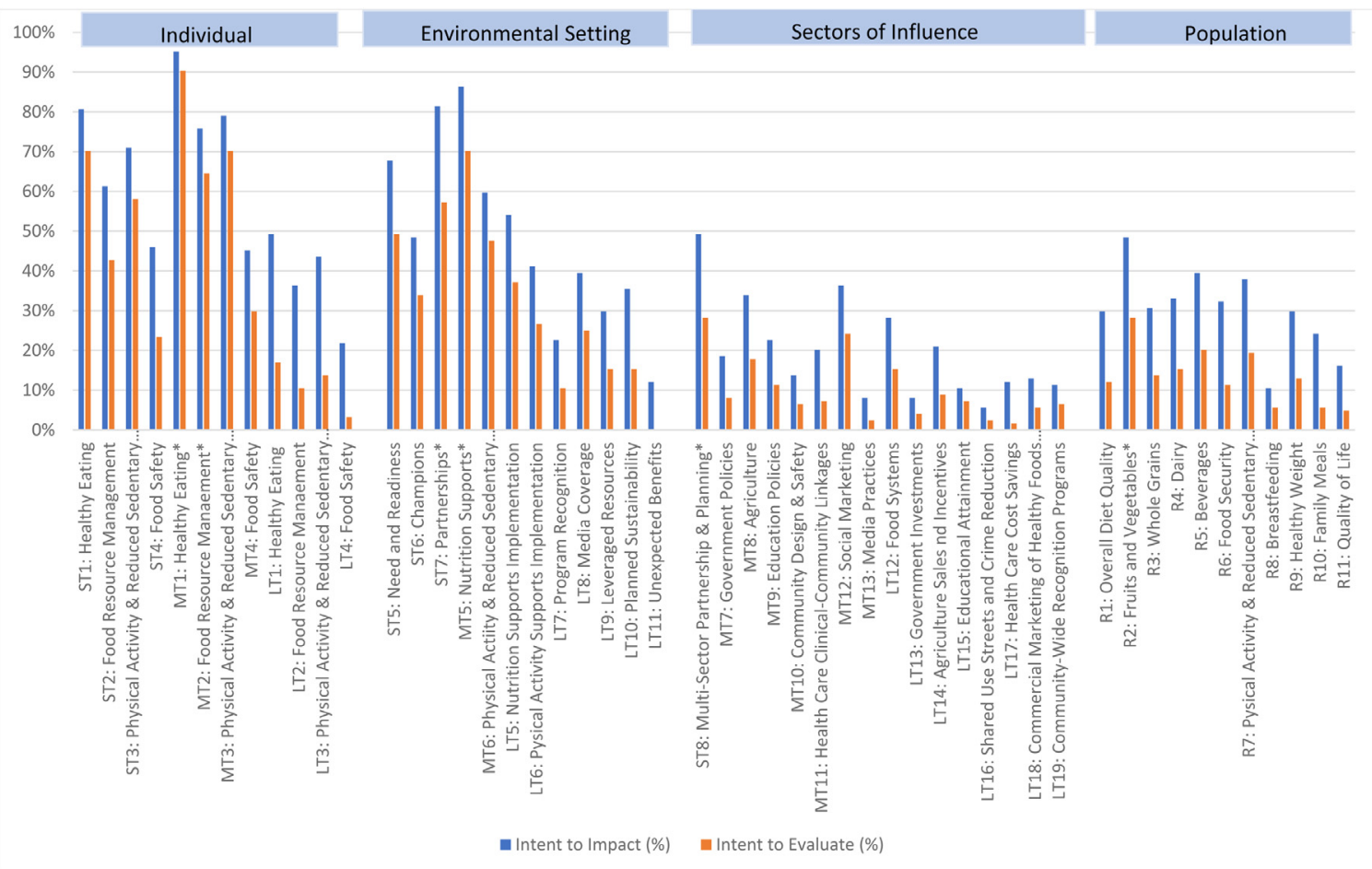

Figure 2. Percentage of SIAs that intend to impact and evaluate each indicator in the Framework $(n=124)$. LT indicates long-term (effectiveness and maintenance); MT, medium-term (behavior changes); SIA, state implementing agency; ST, short-term (readiness and capacity). * Supplemental Nutrition Assistance Program Education (SNAP-Ed) priority indicator. 


\section{Environmental Settings Level}

In this sphere, outcomes are generally defined as the initial adoption of policy, systems or environmental changes in a wide variety of lowresource/high-need settings, ${ }^{5,6}$ followed by longer-term implementation. These outcomes aim to make it easier for people to eat healthily or be active wherever they eat, learn, live, play, shop, or work. Policy, systems, or environmental outcomes are generally aligned with authoritative recommendations for different institutions and verified through objective assessments, such as the Alliance for a Healthier Generation's Scorecard or the Nutrition and Physical Activity Self-Assessment for Child Care. ${ }^{5}$ A lower percentage of SIAs reported intending to impact and evaluate outcomes in this sphere than outcomes for individuals. Very high percentages of SIAs reported intending to impact and evaluate FNS's 2 priority indicators for this sphere: the medium-term nutrition supports (impact $=86 \%$, evaluate $=70 \%$ ) and short-term partnerships (impact $=81 \%$, evaluate $=57 \%$ ).

\section{Sectors of Influence Level}

Outcomes in this sphere generally involve larger-scale, complex, and difficult changes that result only through collaboration among public, nonprofit, and business stakeholders. The purpose is to stimulate and then sustain positive change by entire jurisdictions and organizations in different sectors to help overcome often-longstanding disparities in healthy eating and physical activity. ${ }^{5,6}$ The percent of SIAs intending to impact outcomes in this sphere ranged from $6 \%$ to $49 \%$ and from $2 \%$ to $28 \%$ for intent to evaluate. Once again, the largest percentage of SIAs reported attending to the FNS priority indicator for this sphere, multisector partnerships and planning, with 49\% intending to impact and 28\% planning to evaluate this indicator. About one-third of SIAs planned to impact outcomes related to social marketing and agriculture-related and food system outcomes.

\section{Population Level}

Population-level outcomes are intended to capture changes in behavior among targeted SNAP-Ed population segments as associated with the combined effects and dosage of individual, environmental, and multisector interventions. The intention is to compare dietary and health changes between low-income audiences and the general population and to track reductions in disparities over time. Measurement depends on the availability of national/statewide data sets against which rates among SNAP-Ed population segments and other groups can be compared. When appropriate data sets are not available, indicators are treated as developmental. More population-level indicators were intended to be impacted and evaluated compared with indicators of the sectors of influence (impact range $=10 \%$ $-48 \%$; evaluate range $=5 \%-28 \%$ ). The largest percentage of SIAs intended to impact and evaluate the FNS priority indicator in this section, fruits and vegetables (impact $=48 \%$, evaluate $=28 \%$ ). About one-third of SIAs reported planning to impact 4 other population-wide outcomes: beverages, physical activity and reduced sedentary behavior, dairy, and food insecurity.

\section{DISCUSSION}

The published and gray literature provide abundant evidence of positive SNAP-Ed results throughout the country. ${ }^{10-14}$ However, the difficulty of aggregating data from diverse agencies has hindered the demonstration of national outcomes and impacts of SNAP-Ed. The SNAP-Ed Evaluation Framework addresses this shortfall; it embodies the elements necessary for establishing collective impact-a common agenda, shared measures, mutually reinforcing activities, continuous communication, and strong backbone organizationsall of which offer increased potential for nutrition educators to address stubbornly elusive population improvements. ${ }^{15}$ Although the Framework moves toward the reporting of standardized results, the Census results that represent SNAP-Ed effort across all states help us understand which nutrition education indicators were not yet targeted for intervention by SNAP-Ed SIAs. These were primarily in the outer spheres of the Social Ecological Model ${ }^{7}$ (less than half [48\%] in the environmental setting level, one-third [30\%] in the population level, and even less in the sectors of influence level [20\%]) and when evaluation efforts are needed, that is, most long-term outcomes and outcomes at the sectors of influence and populations level.

A strength of this study was the collaborative nature in which it was conducted. Multiple researchers and evaluators from across the US participated in the planning, piloting, or implementation of the Census. In addition, there was a close collaboration with FNS's 7 SNAP-Ed Regional Coordinators and the SNAP state agencies. The collaborative nature of the study yielded a high response rate $(91 \%)$ and participation from SIAs in all 50 states, which makes the results of the Census highly likely to be generalizable to all SNAP-Ed SIAs and regions of the country. A weakness of the study was the limited participation of the ITOs serving as SIAs. Their limited participation was likely due to the fact that they were newly funded SIAs. Finally, this study was only descriptive in nature; it did not examine whether SIA characteristics predict the use of the Framework. The coauthors intend to explore these types of research questions and look at change over time in the Census results in future publications.

\section{IMPLICATIONS FOR RESEARCH AND PRACTICE}

For nutrition educators more generally, this study offers insights about the very real and large-scale challenges of the work in this field, such as measuring long-term, complex, and multilevel outcomes; gaps and barriers that exist in nutrition education programming and evaluation; technical assistance needs of nutrition education efforts across spheres of influence; and the importance of meaningful collaborations to ensure broader impact across sectors. The Framework and ongoing Census 
results also could provide a template to help track progress by other USDA programs-Special Supplemental Program for Women, Infants and Children, the Expanded Food and Nutrition Education Program, Team Nutrition, and the Gus Schumacher Nutrition Incentive Program (formerly the Food Insecurity Nutrition Incentive Grant Program).

Trends in dietary quality and obesity are generally unfavorable in the US, and there are significant disparities in low-resource populations. ${ }^{16}$ With USDA programs already in place to support high-need groups, having a framework with a set of 51 action-oriented, evidence-based outcome indicators to address the complex conditions that drive these trends reflects significant progress in nutrition education practice. Furthermore, the first-year uptake by so many diverse SNAP-Ed practitioners suggests that it may be feasible to use the Framework more widely as a roadmap to help eliminate disparities between low-resource groups and the general population. That said, this study displayed an uptake in the use of the Framework, namely which indicators SIAs targeted for intervention and evaluated, but it did not aggregate the evaluation results, which would be a next step in demonstrating the national collective impact of SNAP-Ed in eliminating obesityrelated health disparities. Continuing with a nationwide perspective, it is expected that such approaches would not only better characterize the work being conducted through SNAP-Ed, and possibly other nutrition education partners but also lead to closer and more meaningful partnerships at the local, state, and national levels while driving the development of a more robust network of technical assistance, surveillance, reporting, and analysis. This first national snapshot demonstrates the possibility of mobilizing significant and comprehensive efforts to use an evaluation framework, which offers insights about what it will take to begin to demonstrate the collective impact of a national nutrition education program, as it plays a vital role in national nutrition policy and public health.

\section{ACKNOWLEDGMENTS}

The authors wish to sincerely thank all SNAP-Ed implementing agencies and state agencies, without whom this project would not have been possible. The authors also wish to thank Star Morrison, the SNAP-Ed Regional Coordinator for the Mountain Plains Region, for her vision and leadership with conducting the first Census in the Mountain Plains Region, after which the national Census was modeled.

\section{NOTES}

A description of each indicator and some of the corresponding evidencebased measures linked to each indicator can be found at: https://snaped toolkit.org/framework/index/.

\section{REFERENCES}

1. US Department of Agriculture. FY 2019 SNAP-Ed Plan Guidance. Washington, DC: US Department of Agriculture; 2018. https://snaped.fns.usda. gov/snap/Guidance/FY2019SNAPEdPlanGuidanceFULL.pdf. Accessed December 8, 2020.

2. US Department of Health and Human Services, US Department of Agriculture. 2015-2020 Dietary Guidelines for Americans. 8th edition. http://health. gov/dietaryguidelines/2015/guidelines/. Accessed December 8, 2020.

3. US Census Bureau. POV-01. Age and sex of all people, family members and unrelated individuals. https://www. census.gov/data/tables/time-series/ demo/income-poverty/cps-pov/pov01.html\#par_textimage_24. Accessed December 8, 2020.

4. US Government Accountability Office. Nutrition Education: USDA Actions Needed to Assess Effectiveness, Coordinate Programs, and Leverage Expertise. Washington, DC: US Government Accountability Office; 2019. https://www.gao.gov/assets/710/ 700489.pdf. Accessed December 8, 2020.
5. US Department of Agriculture, Food and Nutrition Service. The Supplemental Nutrition Assistance Program Education (SNAP-Ed) Evaluation Framework: nutrition, physical activity, and obesity prevention indicators: interpretive guide to the SNAP-Ed Evaluation Framework. https://snaped.fns. usda.gov/. Accessed December 8, 2020.

6. Naja-Riese A, Keller KJM, Bruno P, et al. The SNAP-Ed Evaluation Framework: demonstrating the impact of a national framework for obesity prevention in low-income populations. Transl Behav Med. 2019;9:970-979.

7. US Department of Health and Human Services, US Department of Agriculture. Chapter 3: Everyone has a role in supporting healthy eating patterns. The social-ecological model. In: 2015-2020 Dietary Guidelines for Americans. 8th edition. https://health.gov/our-work/ food-nutrition/2015-2020-dietaryguidelines/guidelines/chapter-3/socialecological-model/. Accessed December 8, 2020.

8. Harris PA, Taylor R, Thielke R, Payne J, Gonzalez N, Conde JG. Research Electronic Data Capture (REDCap)-a metadata-driven methodology and workflow process for providing translational research informatics support. J Biomed Inform. 2009;42:377-381.

9. Dillman DA. Internet and Mail Surveys: The Tailored Design Method. New York, NY: John Wiley; 2000

10. Contento IR. Nutrition education: linking research, theory, and practice. Asia Pac J Clin Nutr. 2008;17(suppl 1):176179

11. Koszewski W, Sehi N, Behrends D, Tuttle E. The impact of SNAP-ED and EFNEP on program graduates 6 months after graduation. J Ext. 2011;49:1-8.

12. Dannefer R, Abrami A, Rapoport R, Sriphanlop P, Sacks R, Johns M. A mixed-methods evaluation of a SNAP-Ed farmers' market-based nutrition education program. J Nutr Educ Behav. 2015;47:516-525. e1.

13. Rivera RL, Maulding MK, Abbott AR, Craig BA, Eicher-Miller HA. SNAP-Ed (Supplemental Nutrition Assistance Program-Education) increases long-term food security among Indiana households with children in a randomized controlled study. I Nutr. 2016;146:2375-2382. 
14. Hersey JC, Cates SC, Blitstein JL, Williams PA. SNAP-Ed can improve nutrition of low-income Americans across life span (RTI Press publication No. RR-0023-1406). http://www.rti.org/ rtipress. Accessed December 8, 2020.
15. Collective Impact Forum. What is Collective Impact? https://www.collectiveimpactforum.org/what-collectiveimpact. Accessed December 8, 2020.

16. Office of Disease Prevention and Health Promotion. Healthy People
2020: nutrition, physical activity, and obesity. https://www.healthypeople. gov/2020/leading-health-indicators/ 2020-1hi-topics/Nutrition-PhysicalActivity-and-Obesity/data\#NWS-9. Accessed December 8, 2020.

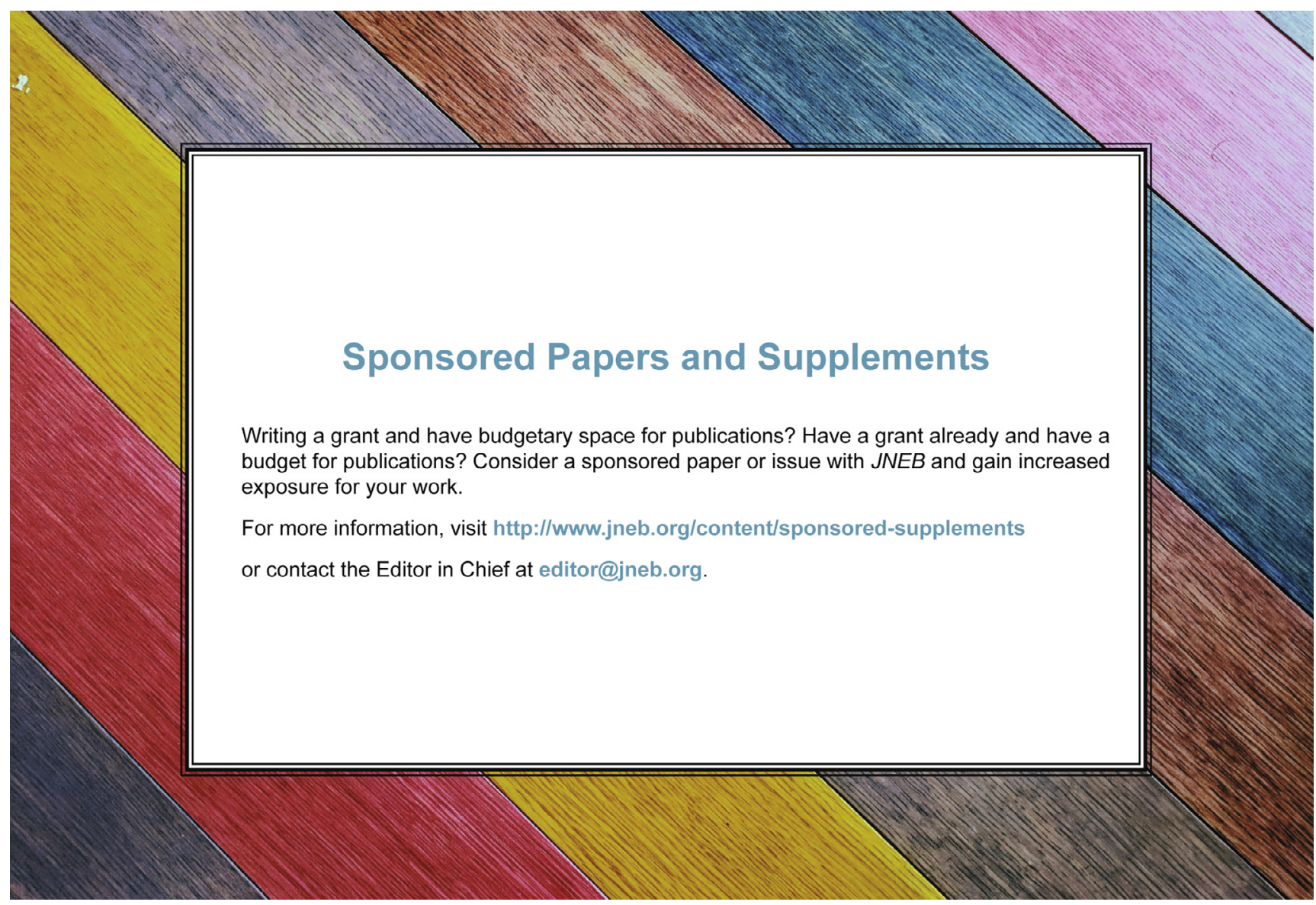

